\title{
Endoscopic ultrasound in diagnosis of solid pancreatic lesions: Elastography or contrast-enhanced harmonic alone versus the combination
}

\section{(ㄷ)(1) $(2) \Theta$}

Authors

Tanyaporn Chantarojanasiri' ${ }^{1}$, Yoshiki Hirooka², Hiroki Kawashima' ${ }^{1}$, Eizaburo Ohno ${ }^{2}$, Takamichi Kuwahara', Takeshi Yamamura $^{2}$, Kohei Funasaka², Masanao Nakamura ${ }^{1}$, Ryoji Miyahara ${ }^{1}$, Masatoshi Ishigami ${ }^{1}$, Osamu Watanabe ${ }^{1}$, Senju Hashimoto $^{3}$, Akihiro Hirakawa ${ }^{4}$, Thawee Ratanachu-ek ${ }^{5}$, Hidemi Goto ${ }^{1}$

Institutions

1 Department of Gastroenterology and Hepatology, Nagoya University Graduate School of Medicine, Nagoya, Japan

2 Department of Endoscopy, Nagoya University Hospital, Nagoya, Japan

3 Department of Liver, Biliary and Pancreas Diseases, Fujita Health University, Toyoake, Japan

4 Center for Advanced Medicine and Clinical Research, Nagoya University Graduate School of Medicine, Nagoya, Japan

5 Department of Surgery, Rajvithi Hospital, Bangkok, Thailand

submitted 23.5.2017

accepted after revision 24.7.2017

Bibliography

DOI https://doi.org/10.1055/s-0043-118829 |

Endoscopy International Open 2017; 05: E1136-E1143

(c) Georg Thieme Verlag KG Stuttgart · New York

ISSN 2364-3722

Corresponding author Yoshiki Hirooka, Department of Endoscopy, Nagoya University Hospital, Nagoya, 65 Tsuruma-cho, Showa-ku, Nagoya City, 466-8550, Japan

Fax: +81 (52) -735-8806

hirooka@med.nagoya-u.ac.jp

\section{ABSTRACT}

Background and study aims Endoscopic ultrasound (EUS) elastography (EUS-E) and contrast-enhanced harmonic EUS (CH-EUS) are useful methods for the diagnosis of pancreatic lesions. This study aims to compare the accuracy of combined EUS-E and CH-EUS with that of EUS-E or $\mathrm{CH}$-EUS alone in the differential diagnosis of pancreatic solid lesions. Patients and methods One hundred thirty-six patients with solid pancreatic lesions underwent EUS with both EUS$\mathrm{E}$ and $\mathrm{CH}$-EUS were included. Diagnoses were classified as adenocarcinoma, neuroendocrine tumor (NET), and inflammatory pseudotumor in 95, 22, and 19 patients, respectively. EUS records in each case were rearranged into 3 groups: EUS-E, CH-EUS, and combination. Each modality was randomly reviewed by 3 reviewers with different levels of clinical experience. Sensitivity, specificity, and accuracy of each modality according to each diagnosis group were evaluated. For the combined diagnosis populations, the proportions of correct diagnoses among the 3 modalities were compared by using the multivariate logistic regression analysis.

Results The accuracies of EUS-E, CH-EUS, and the combination of them were $68.4 \%, 65.4 \%$, and $75.7 \%$, respectively, for adenocarcinoma group; $83.8 \%, 82.4 \%$, and $86.8 \%$ for NET group; $80.1 \%, 78.7 \%$, and $81.6 \%$ for inflammatory pseudotumor group. The multivariate logistic regression analysis for the combined diagnosis populations showed that the proportion of correct diagnoses when EUS-E and $\mathrm{CH}$-EUS were combined was slightly higher than with the other 2 modalities, although the significant differences among them were not observed.

Conclusion EUS-E and CH-EUS combined may improve differential diagnosis of solid pancreatic lesions compared with use of the individual modalities.

\section{Introduction}

Endoscopic ultrasound (EUS) has become an important tool for evaluating the nature of pancreatic diseases. Along with the conventional B mode images, EUS elastography (EUS-E) and contrast-enhanced harmonic EUS (CH-EUS) have been developed to improve the diagnostic accuracy of pancreatic lesions. Elastography measures the tissue deformation after applying dynamic excitation or from cardiac pulsation [1], and translates into different color patterns that reflect tissue stiffness [2,3]. 
Together with conventional B-mode image, elastography increase the sensitivity in the diagnosis of a pancreatic solid mass up to $97 \%$ but with low specificity of $76 \%$ [4]. On the other hand, contrast-enhanced ultrasound characterizes the type of pancreatic lesions based on the hemodynamic properties of the tumor. Together with color Doppler or tissue harmonic imaging [5], it can improve accuracy of diagnosis of pancreatic solid masses, [6, 7] but still with low specificity [6]. For diagnosis of exocrine and endocrine tumor, $\mathrm{CH}$-EUS was able to detect the typical hypervascular pattern of an endocrine tumor with $83.3 \%$ sensitivity but only $60 \%$ specificity [8]. As a result, to improve diagnostic sensitivity and specificity of differential diagnosis of solid pancreatic lesions, we hypothesized that it might be useful to combine these 2 diagnostic modalities. Studies demonstrating the benefit of combinations of endoscopic elastography and contrast-enhanced examination have been limited and results conflicting $[9,10]$.

The aim of this study was to clarify the benefits of combining EUS elastography (EUS-E) and contrast-enhanced harmonicbased EUS (CH-EUS) when compared to EUS-E or CH-EUS as single modalities in differential diagnosis of solid pancreatic lesions.

\section{Patients and methods}

This was a retrospective study of all patients who underwent EUS with both EUS-E and CH-EUS between January 2007 and August 2014 in Nagoya University Hospital, Nagoya, Japan. All patients received the final diagnosis according to the histology of the resected specimen, EUS-guided fine needle aspiration (EUS-FNA), or core needle biopsy. Patients were divided into 3 groups according to pathologic diagnosis: Group 1, adenocarcinoma, which included adenocarcinoma arise from intraductal papillary mucinous neoplasm with predominant solid part; Group 2, pancreatic neuroendocrine tumor (PNET); and Group 3 , inflammatory tumor (autoimmune pancreatitis, mass-forming chronic pancreatitis and granulomatous lesion). Pathology of the PNET group was sub-classified according to revised WHO classification $[11,12]$. At least 6 months of follow up was required for patients diagnosed with chronic pancreatitis by EUS-FNA to exclude the possibility of a false-negative diagnosis of malignancy. This research was in accord with the World Medical Association's Declaration of Helsinki on experiments involving human subjects. Details that might disclose the identity of patients were omitted and the study was approved by the $\mathrm{Na}$ goya University hospital ethics committee.

\section{EUS imaging}

EUS was performed by experienced pancreatobiliary echoendoscopists with more than 4,000 cases of pancreatobiliary EUS (Y.H. and E. O.) or by trainees under supervision by these experienced endoscopists. EG-3670URK, EG-3630UR, EG3630UT and EG-3270UK (Pentax Lifecare Division, Hoya Co., Ltd., Tokyo, Japan) with HI VISION $900^{\circledR}$ or Hitachi Ascendus ${ }^{\circledR}$ (Hitachi Aloka Medical, Ltd., Tokyo, Japan) and GF-UE-260-AL5 (Olympus Co., Tokyo, Japan) with Prosound $\alpha 10^{\circledR}$ (Aloka Medical, Ltd., Tokyo, Japan) or Olympus EU-ME2 PREMIER PLUS ${ }^{\circledR}$
(Olympus Co., Tokyo, Japan) were used as the electronic scanning echoendoscopes for the EUS.

In each case, EUS was initially performed using B-mode with or without tissue harmonic image (THI). Strain elastography was subsequently performed using Real-time Tissue Elastography ${ }^{\circledR}$ (RTE) (Hitachi Aloka Medical, Ltd., Tokyo, Japan) or the ELST mode in EU-ME2 PREMIER PLUS ${ }^{\circledR}$. EUS elastography was performed by using HI VISION 900 as the ultrasound apparatus in 65 patients and using HITACHI ASCENDUS as the ultrasound apparatus in another 70 patients. The Region of Interest (ROI) was set to include the area of solid tumors and surrounding soft tissues. Relative elasticity of tissue was expressed as colors: blue (hard tissue), green (average) and red (soft). During the procedure, elastography images were manually selected by the operator and stored as videos and still color images in the ultrasound apparatus as avi and jpeg files, respectively. The contrast study was performed by using one vial of Sonazoid ${ }^{\circledR}$ $(16 \mu \mathrm{l}$ as perfluorobutane, Daiichi Sankyo, Tokyo, Japan) suspended in $2 \mathrm{~mL}$ of sterile water at a dose of $0.015 \mathrm{~mL} / \mathrm{kg}$ injected via a $22 \mathrm{G}$ needle catheter inserted in the antecubital vein and flushed with at least $10 \mathrm{~mL}$ of normal saline. In cases using EG-3670URK, EG-3630UR, EG-3630UT and EG-3270UK with HI VISION $900^{\circledR}$ or Hitachi Ascendus ${ }^{\circledR}, \mathrm{CH}$-EUS was performed under a wide-band pulse inversion method and the mechanical index was automatically set at 0.16 to 0.23 in accordance with the focal point. In cases using GF-UE-260-AL5 with Prosound $\alpha 10^{\circledR}$ or Olympus EU-ME2 PREMIER PLUS ${ }^{\circledR}$, the extended pure harmonic detection method (ExPHD) was used, and the mechanical index was set at 0.25 . Image observation was acquired using contrast-enhanced harmonic imaging continuously for 5 minutes. Video records were made at 1,3 and 5 minutes after injection. In cases using Hitachi Ascendus ${ }^{\circledR}$ and Prosound $\alpha 10^{\circledR}$ as the ultrasonic apparatuses, time intensity curves were calculated using the installed software in the machine. Serial still images were recorded with labelled time sequence or the video records of the contrast enhanced study at each time point after contrast injection. TIC was performed using digital data stored on a hard disk of the ultrasound apparatus. Regions of interest (ROI) with maximal possible size were selected at the lesion and at surrounding pancreatic parenchyma. Echointensity after contrast injection in both ROI was calculated by the software installed in the machine. EUS images and video were taken during the examination and all records were rearranged into 3 categories (as described below) and sent for blind revision.

\section{Image interpretation}

Stored images of the same patients were divided into 3 imaging categories: B-mode images (including color Doppler and tissue harmonic imaging) with EUS-E; B-mode images with $\mathrm{CH}$-EUS including time intensity curve analysis (TIC); and B-mode images with both EUS-E and $\mathrm{CH}$-EUS (combination). Patient order ran according to the timing of EUS examination, and in each case, each imaging category was randomly interpreted by 3 reviewers with different levels of EUS experience ( 2 experts and 1 trainee). All reviewers were blinded to patient clinical presentation and other imaging findings. All examinations were performed 


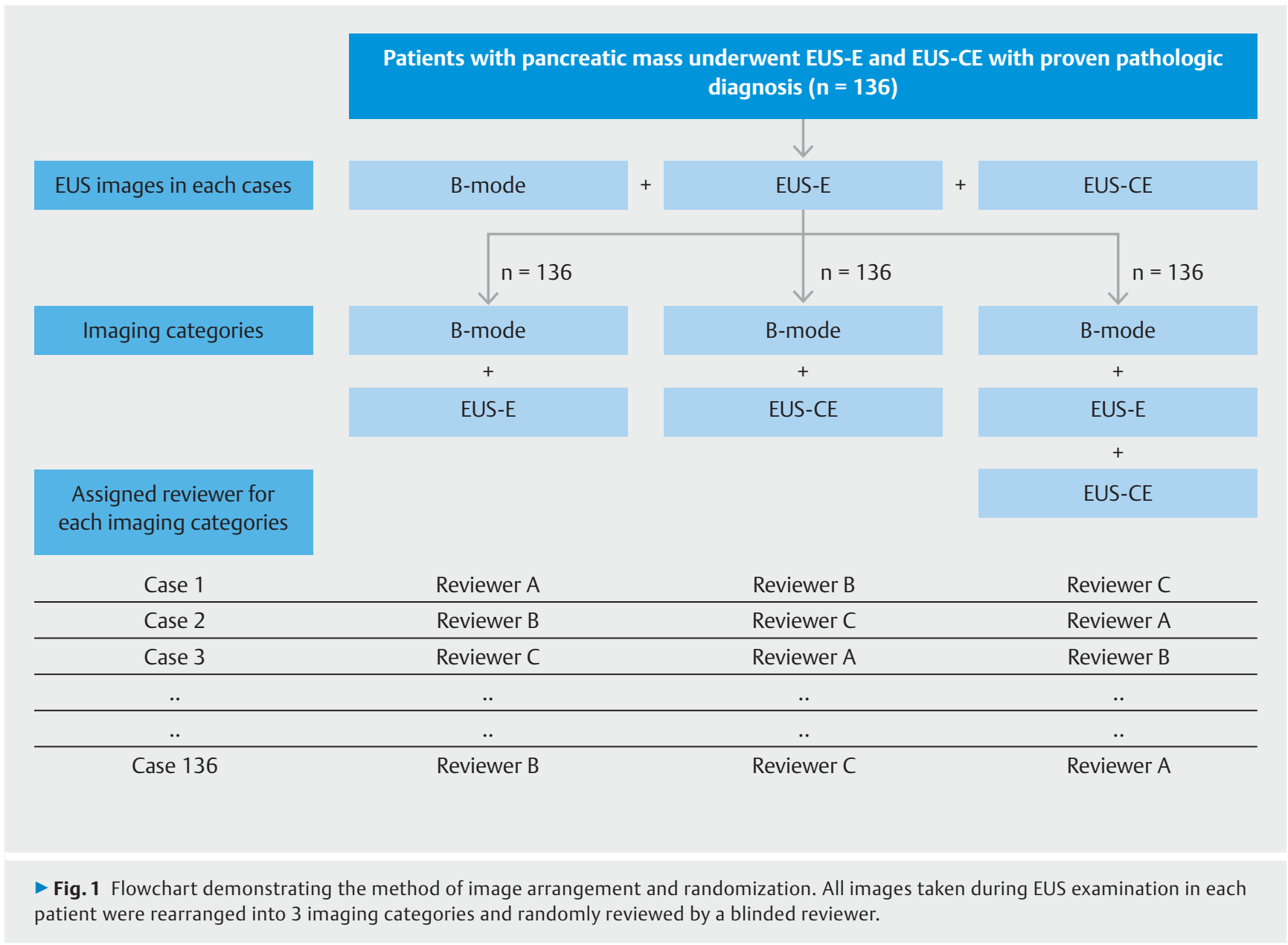

more than 6 months before image interpretation to reduce reviewer bias. - Fig. 1 illustrates the method of randomization.

Interpretation of images from EUS-E was performed using the elastic score, as proposed by Giovannini [7]: score 1, distortion for entire low echo area (normal pancreas); score 2, no distortion on low echo area even for a part (chronic pancreatitis); score 3 , distortion at the edge of low echo area (small adenocarcinoma); score 4, no distortion for entire low echo area (endocrine tumor); score 5, no distortion on low echo area and surrounding (advanced adenocarcinoma). Interpretation of $\mathrm{CH}$-EUS was performed based on multiple serial images of CE-EUS together with labelled time sequence or video records after contrast injection. $\mathrm{CH}$-EUS imaging was interpreted into 3 patterns in a report by Matsubara [13]: heterogeneously hypovascular enhancing mass (pancreatic adenocarcinoma), rapid-stained hypervascular mass (endocrine tumor), and homogeneously isovascular mass (inflammatory pseudotumor) ( $\triangleright$ Fig. 2). After revision, all reviewers gave the presumptive diagnosis according to the 3 diagnostic categories which were compared to the pathologic diagnosis as the gold standard. When EUS-E and CE-EUS were not correlated, the decision about diagnosis was dependent upon each reviewer's personal experience.

\section{Statistical analysis}

Sensitivity, specificity, and accuracy of EUS-E, CH-EUS and the combination in each pathologic diagnostic group were calculated using pathological diagnosis as the gold standard. For the combined 3 diagnosis populations, multivariate logistic regression analysis was performed with adjustment for the effects of the 3 reviewers to compare the proportion of correct diagnoses among the 3 modalities. A two-sided $P<0.05$ was considered to be statistically significant. The statistical software SPSS ver. 23 (SPSS, Inc, Chicago, IL) was used for all statistical analyses.

\section{Results}

In total, 242 patients who underwent both EUS-E and $\mathrm{CH}$-EUS were recruited. However, 106 patients were excluded due to lack of or non-diagnostic pathology results, other diagnosis not classified in our study, presence of predominant cystic components, or loss to follow-up. The remaining 136 patients, including 54 females (39.7\%) and 82 males (60.3\%), aged 28 to and 83 years (mean $65.1 \pm 10.2$ ), were eligible for image analysis. Diagnoses were made based on surgical specimens in 91 patients (66.9\%), EUS-FNA in 41 patients (30.1\%), and endoscopic, percutaneous and laparoscopic biopsy in 4 patients 

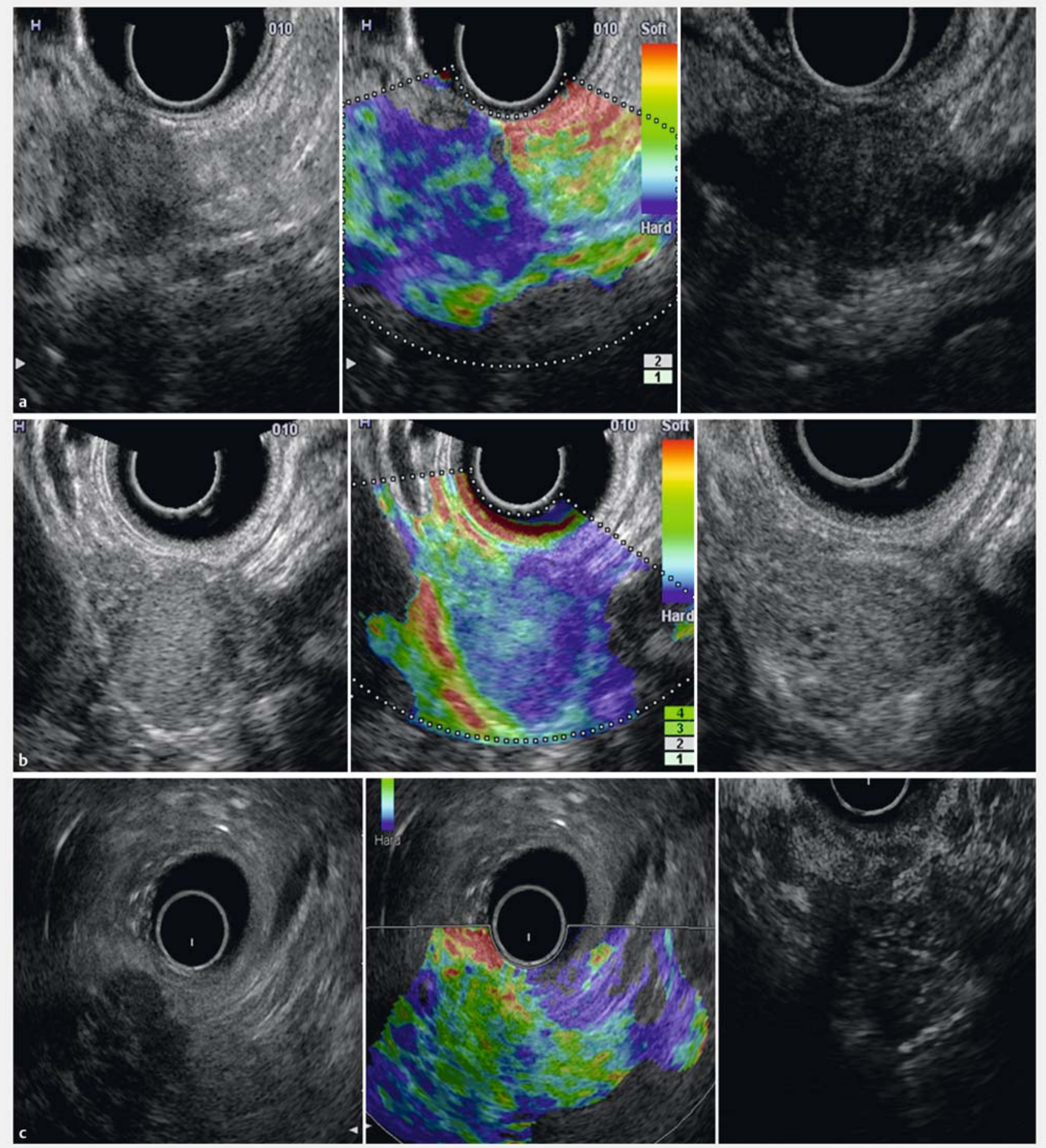

- Fig. 2 EUS images in B-mode, EUS-E and CH-EUS in each diagnostic modality. a Group 1 (carcinoma) case showing an elastography score of 5 and a hypovascular pattern on contrast-enhanced imaging. b Group 2 (PNET) case showing an elastography score of 4 and a hypervascular pattern on contrast-enhanced imaging. c Group 3 (inflammatory) case showing an elastography score of 2 and an isovascular pattern on contrast-enhanced imaging.

(2.9\%). There were 95 patients $(69.9 \%)$ in the adenocarcinoma group, 22 patients (16.2\%) in the PNET group, and 19 patients $(14 \%)$ in the inflammatory pseudotumor group. Among those with PNET, there were 3 patients classified as G2 and 19 patients classified as G1 according to ENETS classification by re- vised WHO classification 2016 [12]. Each reviewer gave the diagnosis for 45 to 47 patients in each imaging set (EUSE, CE-EUS and combination). The number of still images provided for the revision ranged from 25 to 239 images, with a median of 84, 107 and 124 for the still images in EUS-E, CE-EUS and com- 
- Table 1 Demographic data.

\begin{tabular}{|c|c|c|c|c|}
\hline & \multicolumn{3}{|c|}{ Diagnostic categories* } & \multirow[t]{2}{*}{ Total $(n=136)$} \\
\hline & Group $1(n=95)$ & Group $2(n=22)$ & Group $3(n=19)$ & \\
\hline Mean Age (range) & $67.6(38-83)$ & $56.4(28-76)$ & $62.8(35-80)$ & $65.1(28-83)$ \\
\hline Gender (female) (\%) & $38(39.6 \%)$ & $16(72.7 \%)$ & $4(21.1 \%)$ & $54(39.7 \%)$ \\
\hline Size of tumor (mm) (range) & $28.9(4-110)$ & $23.2(8-51)$ & $25.8(8-59)$ & $27.6(4-110)$ \\
\hline \multicolumn{5}{|l|}{ Tumor location } \\
\hline - Head & 62 & 8 & 13 & 83 \\
\hline - Body & 26 & 7 & 5 & 38 \\
\hline - Tail & 7 & 7 & 1 & 15 \\
\hline \multicolumn{5}{|l|}{ Diagnostic method } \\
\hline - surgery & $64(66.7 \%)$ & $20(90.1 \%)$ & $8(42.1 \%)$ & 91 \\
\hline - FNA & $29(30.2 \%)$ & $1(4.5 \%)$ & $11(57.9 \%)$ & 41 \\
\hline - biopsy & $3(3 \%)$ & $1(4.5 \%)$ & 0 & 4 \\
\hline Diagnosis & $\begin{array}{l}\text { - } \text { adenocarcinoma } 87 \text { (91.6\%) } \\
\text { - } \text { adenosquamous carcinoma } 2(2.1 \%) \\
\text { - carcinoma arised from intraductal } \\
\text { papillary mucinous carcinoma } 6 \text { (6.3\%) }\end{array}$ & - PNET 22 & $\begin{array}{l}\text { - } \text { AIP } 12(63.2 \%) \\
\text { - } \text { mass forming chronic } \\
\text { pancreatitis } 4(21.1 \%) \\
\text { - } \text { granuloma } 3(15.8 \%)\end{array}$ & \\
\hline
\end{tabular}

- Table 2 Sensitivity, specificity and accuracy of each modality according to each diagnosis group.

\begin{tabular}{|c|c|c|c|c|}
\hline Diagnostic category & Diagnostic modality & Sensitivity $(95 \% \mathrm{Cl})$ & Specificity (95\% Cl) & Accuracy $(95 \% \mathrm{Cl})$ \\
\hline \multirow{3}{*}{$\begin{array}{l}\text { Group } 1 \\
\text { Adenocarcinoma }\end{array}$} & EUS-E & $67.4 \%(57.3-76.5)$ & $70.7 \%(60.7-79.4)$ & $68.4 \%(58.3-77.4)$ \\
\hline & CH-EUS & $66.3 \%(56.1-75.5)$ & $63.4 \%(53.2-72.8)$ & $65.4 \%(55.2-74.6)$ \\
\hline & Combination & $71.6 \%(61.7-80.2)$ & $85.4 \%(76.9-91.7)$ & $75.7 \%(66.1-83.8)$ \\
\hline \multirow{3}{*}{$\begin{array}{l}\text { Group } 2 \\
\text { PNET }\end{array}$} & EUS-E & $63.6 \%(53.3-73)$ & $87.7 \%(79.5-93.5)$ & $83.8 \%(75.1-90.4)$ \\
\hline & CH-EUS & $63.6 \%(53.3-73)$ & $86 \%(77.6-92.1)$ & $82.4 \%(73.5-89.3)$ \\
\hline & Combination & $77.3 \%(67.8-85.1)$ & $88.6 \%(80.6-94.2)$ & $86.8 \%(78.5-92.8)$ \\
\hline \multirow{3}{*}{$\begin{array}{l}\text { Group } 3 \\
\text { Inflammatory }\end{array}$} & EUS-E & $63.2 \%(52.9-72.7)$ & $82.9 \%(74-89.7)$ & $80.1 \%(70.9-87.5)$ \\
\hline & CH-EUS & $47.4 \%(37.3-57.7)$ & $83.8 \%(75.1-90.4)$ & $78.7 \%(69.3-86.3)$ \\
\hline & Combination & $68.4 \%(58.3-77.4)$ & $83.8 \%(75.1-90.4)$ & $81.6 \%(72.5-88.7)$ \\
\hline
\end{tabular}

bined, respectively. Demographic data are shown in $>$ Table 1. Lesions were located on the pancreatic head in 83 patients (61.0\%), on the pancreatic body in 38 patients $(27.9 \%)$ and on the pancreatic tail in 15 patients $(11.0 \%)$ with size ranging from 4 to $110 \mathrm{~mm}$ (median $25 \mathrm{~mm}$ ).

The sensitivity, specificity, and accuracy of each modality in the 3 diagnostic groups are shown in > Table 2. CE-EUS's sensitivity was generally lower than that for other modalities, especially in diagnosis of inflammatory lesions. The proportion of correct diagnosis for EUS-E, CE-EUS, and the combination of them were $66.2 \%(95 \% \mathrm{Cl} 57.6-74.1), 63.2 \%(95 \% \mathrm{Cl} 54.5-$ $71.3)$ and $72.1 \%(95 \% \mathrm{Cl} 63.7-79.4)$, respectively. According to results of multivariate logistic regression analysis, the odds ratio and its $95 \%$ confidence interval $(\mathrm{Cl})$ of the combination group relative to the $\mathrm{CH}$-EUS and EUS-E were 1.50 (0.90 to 2.50) and 1.32 ( 0.79 to 2.22 ), respectively ( $\triangleright$ Table 3 ). The tendency of improvement in terms of correct diagnosis by combining $\mathrm{CH}$ EUS and EUS-E was observed, but there were no statistically significant differences among them. Furthermore, there was no statistically significant difference in diagnostic correctness among the reviewers ( $\downarrow$ Table 3 ). However, in subgroup analysis for the 3 diagnostic populations, the correctness between the trainee (Reviewer A) and the experts (Reviewer C) became significant in Group 3 ( $>$ Table 3 ). The diagnosis in Group 3 using EUS-E was $100 \%$ accurate for reviewers $A$ and $B$ (the experts), but accuracy was only $22 \%$ for reviewer $C$ (the trainee). 
Table 3 Comparison of proportion of correct diagnosis among three modalities based on multivariate logistic regression analysis.

\begin{tabular}{|c|c|c|c|c|c|c|c|c|}
\hline \multicolumn{2}{|c|}{ Categories } & \multicolumn{3}{|c|}{ Comparison } & \multirow{2}{*}{$\begin{array}{l}\text { Odds ratio (OR) } \\
1.50\end{array}$} & \multicolumn{2}{|c|}{$95 \% \mathrm{Cl}$} & \multirow{2}{*}{$\begin{array}{l}P \text { value } \\
0.1247\end{array}$} \\
\hline All diagnoses & Modality & Combination & vs & CE-EUS & & 0.90 & 2.50 & \\
\hline & & Combination & vs & EUS-E & 1.32 & 0.79 & 2.22 & 0.2933 \\
\hline & Reviewer & B & vs & A & 0.81 & 0.48 & 1.36 & 0.4164 \\
\hline & & C & vs & A & 0.63 & 0.38 & 1.05 & 0.0736 \\
\hline \multirow[t]{4}{*}{ Group 1} & \multirow[t]{2}{*}{ Modality } & Combination & vs & CE-EUS & 1.28 & 0.69 & 2.37 & 0.4402 \\
\hline & & Combination & vs & EUS-E & 1.24 & 0.67 & 2.31 & 0.4987 \\
\hline & \multirow[t]{2}{*}{ Reviewer } & B & vs & A & 0.63 & 0.34 & 1.19 & 0.1539 \\
\hline & & C & vs & A & 0.63 & 0.34 & 1.19 & 0.1543 \\
\hline \multirow[t]{4}{*}{ Group 2} & \multirow[t]{2}{*}{ Modality } & Combination & vs & CE-EUS & 1.99 & 0.52 & 7.63 & 0.3171 \\
\hline & & Combination & vs & EUS-E & 1.99 & 0.52 & 7.63 & 0.3171 \\
\hline & \multirow[t]{2}{*}{ Reviewer } & B & vs & A & 1.13 & 0.31 & 4.16 & 0.8552 \\
\hline & & C & vs & A & 1.13 & 0.31 & 4.16 & 0.8552 \\
\hline \multirow[t]{4}{*}{ Group 3} & \multirow[t]{2}{*}{ Modality } & Combination & vs & CE-EUS & 3.04 & 0.68 & 13.64 & 0.1461 \\
\hline & & Combination & vs & EUS-E & 0.86 & 0.19 & 3.89 & 0.8458 \\
\hline & \multirow[t]{2}{*}{ Reviewer } & B & vs & A & 1.93 & 0.42 & 8.81 & 0.3942 \\
\hline & & C & vs & A & 0.23 & 0.05 & 0.98 & 0.0476 \\
\hline
\end{tabular}

- Table 4 Sensitivity, specificity and accuracy of each modality when the least experienced reviewer was excluded.

\begin{tabular}{|c|c|c|c|c|}
\hline Diagnostic category & Diagnostic modality & Sensitivity (95\% CI) & Specificity (95\% CI) & Accuracy $(95 \% \mathrm{Cl})$ \\
\hline \multirow{3}{*}{$\begin{array}{l}\text { Group } 1 \\
\text { Adenocarcinoma }\end{array}$} & EUS-E & $95.7 \%(89.5-98.8)$ & $53.5 \%(43.2-63.5)$ & $74.7 \%(65.0-82.9)$ \\
\hline & CH-EUS & $66.2 \%(56-75.4)$ & $42.9 \%(33.0-53.2)$ & $64.4 \%(54.2-73.7)$ \\
\hline & Combination & $92 \%(84.8-96.5)$ & $61 \%(50.7-70.6)$ & $78 \%(68.6-85.7)$ \\
\hline \multirow{3}{*}{$\begin{array}{l}\text { Group } 2 \\
\text { PNET }\end{array}$} & EUS-E & $57.9 \%(47.6-67.7)$ & $93.1 \%(86.1-97.3)$ & $85.7 \%(77.2-92.0)$ \\
\hline & CH-EUS & $46.7 \%(36.6-57.0)$ & $93.3 \%(86.4-97.4)$ & $85.6 \%(77.1-91.9)$ \\
\hline & Combination & $66.7 \%(56.5-75.8)$ & $94.5 \%(88.1-98.1)$ & $89 \%(81.2-94.4)$ \\
\hline \multirow{3}{*}{$\begin{array}{l}\text { Group } 3 \\
\text { Inflammatory }\end{array}$} & EUS-E & $41.7 \%(31.9-52.0)$ & $100 \%$ & $84.6 \%(75.9-91.1)$ \\
\hline & CH-EUS & $35 \%(25.7-45.2)$ & $88.6 \%(80.6-94.2)$ & $76.7 \%(67.1-84.6)$ \\
\hline & Combination & $43.5 \%(33.6-53.8)$ & $95.5 \%(89.4-98.6)$ & $82.4 \%(73.5-89.3)$ \\
\hline
\end{tabular}

After excluding the least experienced endosonographer, sensitivity of EUS-E improved in Group 1, and specificity increased for all modalities in Groups 2 and 3 ( $>$ Table 4). Multivariate analysis after exclusion of data from the least experienced endosonographer did not show any significant difference in diagnostic populations with all modalities ( $>$ Table 5). Factors that may influence diagnosis of ductal adenocarcinoma were analyzed. Tumor size and location and the machine used (HI VISION 900 and HITACHI ASCENDUS), as well as the number of images, video and presence of TIC were did not differ for those who received correct versus incorrect diagnoses of adenocarcinoma.

\section{Discussion}

EUS elastography and contrast-enhanced EUS have been reported to improve sensitivity pf diagnosis of solid pancreatic lesions. However, performing both modalities in every case require a lot of equipment, which may not be available in some centers. In our study, we compared diagnostic ability of EUS elastography and contrast-enhanced EUS, both as individual modalities and in combination. We divided patients into 3 diagnostic groups that had different patterns of contrast enhancement [13]. Other uncommon types of pancreatic tumor, such as solid pseudopapillary neoplasm and acinar cell carcinoma, were not included due to their small number and limited de- 
- Table 5 Comparison of proportion of correct diagnosis among 3 modalities based on multivariate logistic regression analysis without data from the trainee (reviewer C).

\begin{tabular}{|c|c|c|c|c|c|c|c|c|}
\hline \multicolumn{2}{|l|}{ Categories } & \multicolumn{3}{|l|}{ Comparison } & \multirow{2}{*}{$\begin{array}{l}\text { Odds ratio (OR) } \\
1.68\end{array}$} & \multicolumn{2}{|c|}{$95 \% \mathrm{Cl}$} & \multirow{2}{*}{$\begin{array}{l}P \text { value } \\
0.1100\end{array}$} \\
\hline All diagnoses & Modality & Combination & vs & CE-EUS & & 0.89 & 3.17 & \\
\hline & & Combination & vs & EUS-E & 1.08 & 0.56 & 2.07 & 0.8240 \\
\hline & Reviewer & B & vs & A & 0.81 & 0.48 & 1.36 & 0.4161 \\
\hline \multirow[t]{3}{*}{ Group 1} & \multirow[t]{2}{*}{ Modality } & Combination & vs & CE-EUS & 1.29 & 0.59 & 2.79 & 0.5228 \\
\hline & & Combination & vs & EUS-E & 1.22 & 0.57 & 2.64 & 0.6098 \\
\hline & Reviewer & B & vs & A & 0.63 & 0.34 & 1.19 & 0.1545 \\
\hline \multirow[t]{3}{*}{ Group 2} & \multirow[t]{2}{*}{ Modality } & Combination & vs & CE-EUS & 2.16 & 0.43 & 10.91 & 0.3523 \\
\hline & & Combination & vs & EUS-E & 1.38 & 0.28 & 6.74 & 0.6883 \\
\hline & Reviewer & B & vs & A & 1.06 & 0.29 & 3.92 & 0.9334 \\
\hline \multirow[t]{3}{*}{ Group 3} & \multirow[t]{2}{*}{ Modality } & Combination & vs & CE-EUS & 2.72 & 0.51 & 14.44 & 0.2395 \\
\hline & & Combination & vs & EUS-E & 0.11 & 0.004 & 2.85 & 0.1843 \\
\hline & Reviewer & B & vs & A & 2.25 & 0.46 & 11.05 & 0.3187 \\
\hline
\end{tabular}

scription of their contrast-enhancement and/or elastography patterns. The number of patients in each age group differed according to the differences in disease prevalence. However, inflammatory pseudotumor prevalence was much lower than the usual prevalence due to the lack of tissue diagnosis in many cases.

Studies of the efficacy of combining EUS elastography and contrast-enhanced EUS are limited. The study by Saftoiu [9] comparing contrast-enhanced power Doppler, real-time elastography and the combination of both modalities in differential diagnosis of pancreatic adenocarcinoma and chronic pancreatitis showed sensitivities of $90.9 \%, 84.8 \%$ and $75.8 \%$, respectively. Another study by Hocke [10] using elastography, low-mechanical index CE-EUS and high-mechanical index CE-EUS in differential diagnosis of pancreatic cancer and chronic pancreatitis showed sensitivities of $94.7 \%, 84.2 \%$ and $89.5 \%$, respectively. When combined EUS elastography, low-mechanical index CE-EUS and high-mechanical index EUS was used, the sensitivity increased up to $94.7 \%$. In this report, this whole combination is not superior to the combination of B-mode with contrast-enhanced modalities. Unlike our study, these 2 reports were mainly based on contrast-Doppler mode and evaluated only differential diagnosis of pancreatic adenocarcinoma and chronic pancreatitis. Another recent study using CE-EUS and EUS elastography in focal pancreatic mass with negative EUSFNA showed a promising result in differential diagnosis of benign and malignant lesions [14]. Even though that study also used contrast-enhanced harmonic imaging, it evaluated only those with chronic pancreatitis and pancreatic cancer. These differences in study population, method of image analysis (qualitative versus semiquantitative), type of image analyzed (real time video images versus off-line stored still images) and experience of the image reviewer might contribute to the different results in our study.
We conducted a study comparing the diagnostic efficacy of EUS-E, CH-EUS and the combination in a single-blinded fashion. Since this study reviewed the data over a 7-year period, different ultrasound apparatuses equipped with different software were used depending on the machine available at each time point. Using the different software for image interpretation could lead to significant heterogeneity of the study. As a result, we used qualitative analysis to unify elastography interpretation. Regarding the type of elastography interpretation, data from a meta-analysis showed that EUS-E in differential diagnosis of pancreatic adenocarcinoma and inflammatory mass with use of the color pattern as the diagnostic standard had a pooled sensitivity of 0.99 and pooled specificity of 0.76 , compared with pooled sensitivity of 0.92 and pooled specificity of 0.68 for use of the hue histogram [15]. The data confirmed that color pattern diagnosis that provides quantitative information may be preferable to the more qualitative method. We also performed an analysis comparing elastography results with use of the older HIVISION 900 and the newer ASCENDUS system but did not found any difference in accuracy of diagnosis.

Diagnosis of solid pancreatic lesions was classified into 3 groups of lesion of different natural histories and well-known pattern of contrast-enhancement and elastography. Comparison using multivariate analysis for correct proportion demonstrated favorable trends for use of combination modalities in all diagnostic groups, but the results did not reach statistical significance. That is similar to the report by Hocke [10], which did not demonstrate the benefit of combining EUS-E and CEEUS. However, according to our data, the combination may be beneficial in cases for which inflammatory pseudotumor is the main differential diagnosis or EUS is performed by a less experienced endosonographer. Sensitivity, specificity, NPV, PPV, and accuracy in our study differed from that in other studies mentioned earlier, which may be because of several reasons. First, in our study, the reviewer interpreted the results based on the 
off-line, stored images, which is different from real-time or video-recorded interpretation. However, because we used the same interpretation method for all diagnostic modalities, that limitation should not interfere with the final result. Second, our study used quantitative methods of interpretation, which are different from those in other reports. Moreover, the image reviewers in our study also included a trainee, which differed from most studies in which it was performed only by experts. In our data, the differences between experts and trainees decreased with use of combined modalities. After excluding the least experienced endosonographer, there was no difference among EUS-E, CE-EUS and combination in differential diagnosis of solid pancreatic lesions.

Our study has several strong points. We performed the single-blinded study in 136 patients, which is a much higher number of patients than in other studies, and included various types of pancreatic mass lesions. Pathological results were the gold standard for diagnosis in all cases. In addition, we also included a non-expert reviewer, which makes our results a better representation of the real-life situation. Our technique of EUS-E and CE-EUS interpretation was color pattern analysis, which was simple and did not require special software calculations. That makes our study applicable to many institutions in which special image processors or software are not available. Moreover, our study involved both experienced and inexperienced endosonographers, which might be a better representation of the real-world situation.

On the other hand, there were several limitations. First, this study was based on retrospective case reviews that not only analyzed the video but also still images, which may decrease diagnostic sensitivity and specificity, especially for interpretation of CE-EUS, in which many serial images taken together with the labelled time sequence were used as the substitute. However, we did not find a significant difference between accuracy and the number of video images and that limitation may not affect our comparison between modalities, which all used the same method. Second, the study was performed over a 7-year period, during which different equipment and technologies were available. Using the different kinds of ultrasound processors may bring about some bias, specifically, different probes may show different images. However, the essence of images was the same and we compared the diagnosis of the same case. Moreover, some of the examinations were performed by the reviewers, which could lead to significant recall bias despite a severalmonth interval between examination and study. Because the prevalence of diseases in each group differed, the number of cases in each diagnostic group was small and uneven, which resulted in a low power of the study.

\section{Conclusion}

In summary, EUS elastography and contrast-enhanced EUS combined improved sensitivity, specificity and accuracy of diagnosis of solid pancreatic lesions compared with use of the modalities individually. However, that improvement did not reach statistical significance.
Competing interests

None

References

[1] Hirooka Y, Kuwahara T, Irisawa A et al. JSUM ultrasound elastography practice guidelines: pancreas. J Medical Ultrasonics 2015; 42: 151 174

[2] Janssen J, Schlorer E, Greiner L. EUS elastography of the pancreas: feasibility and pattern description of the normal pancreas, chronic pancreatitis, and focal pancreatic lesions. Gastrointest Endosc 2007; 65: $971-978$

[3] Kuwahara T, Hirooka Y, Kawashima $\mathrm{H}$ et al. Quantitative diagnosis of chronic pancreatitis using EUS elastography. J Gastroenterol 2017; 52: $868-874$

[4] Hu DM, Gong TT, Zhu Q. Endoscopic ultrasound elastography for differential diagnosis of pancreatic masses: a meta-analysis. Dig Dis Sci 2013; 58: $1125-1131$

[5] Hirooka Y, Itoh A, Kawashima $\mathrm{H}$ et al. Contrast-enhanced endoscopic ultrasonography in digestive diseases. J Gastroenterol 2012; 47: $1063-1072$

[6] Fusaroli P, Spada A, Mancino MG et al. Contrast harmonic echoendoscopic ultrasound improves accuracy in diagnosis of solid pancreatic masses. Clin Gastroenterol Hepatol 2010; 8: 629-634 (e621622)

[7] Giovannini M, Hookey LC, Bories E et al. Endoscopic ultrasound elastography: the first step towards virtual biopsy? Preliminary results in 49 patients Endoscopy 2006; 38: $344-348$

[8] Serra C, Felicani C, Mazzotta E et al. Contrast-enhanced ultrasound in the differential diagnosis of exocrine versus neuroendocrine pancreatic tumors. Pancreas 2013; 42: 871-877

[9] Saftoiu A, lordache SA, Gheonea DI et al. Combined contrast-enhanced power Doppler and real-time sonoelastography performed during EUS, used in the differential diagnosis of focal pancreatic masses (with videos). Gastrointest Endosc 2010; 72: 739- 747

[10] Hocke M, Ignee A, Dietrich CF. Advanced endosonographic diagnostic tools for discrimination of focal chronic pancreatitis and pancreatic carcinoma-elastography, contrast enhanced high mechanical index (CEHMI) and low mechanical index (CELMI) endosonography in direct comparison. Z Gastroenterol 2012; 50: 199-203

[11] Bosman FT, World Health Organization, International Agency for Research on Cancer. WHO classification of tumours of the digestive system. 4th ed. Lyon: International Agency for Research on Cancer; 2010: 417

[12] Ito T, Hijioka S, Masui T et al. Advances in the diagnosis and treatment of pancreatic neuroendocrine neoplasms in Japan. J Gastroenterol 2016; 52: 9-18

[13] Matsubara H, Itoh A, Kawashima $\mathrm{H}$ et al. Dynamic quantitative evaluation of contrast-enhanced endoscopic ultrasonography in the diagnosis of pancreatic diseases. Pancreas 2011; 40: 1073-1079

[14] Iordache S, Costache MI, Popescu CF et al. Clinical impact of EUS elastography followed by contrast-enhanced EUS in patients with focal pancreatic masses and negative EUS-guided FNA. Med Ultrason 2016; $18: 18-24$

[15] Li X, Xu W, Shi J et al. Endoscopic ultrasound elastography for differentiating between pancreatic adenocarcinoma and inflammatory masses: a meta-analysis. World J Gastroenterol 2013; 19: 6284-6291 